\title{
(2) OPEN ACCESS \\ Implementation of a novel digital diagnostic tool to support the assessment of respiratory disease in a COVID-19 fever clinic
}

\author{
Andrew Ladhams (D) ,' Shrawan Patel (D) , ${ }^{2}$ Mathew Çetin (D) ${ }^{1}$
}

\begin{abstract}
${ }^{1}$ Health Hub Doctors Morayfield, Morayfield, Queensland, Australia

${ }^{2}$ Strategy Health, New York City, New York, USA
\end{abstract}

\section{Correspondence to} Dr Shrawan Patel, Strategy Health, New York City, New York, USA; Shrawan.patel@ doctors.net.uk

$\mathrm{AL}$ and SP are joint first authors.

Received 12 January 2021 Accepted 18 December 2021 Published Online First

12 January 2022

\section{Check for updates}

\section{(C) Author(s) (or their} employer(s)) 2022. Re-use permitted under CC BY-NC. No commercial re-use. See rights and permissions. Published by BMJ.

To cite: Ladhams $\mathrm{A}$, Patel S, Çetin M. BMJ Innov 2022;8:55-59.

\section{INTRODUCTION}

Following its first detection in Wuhan, China, in December $2019,{ }^{1}$ the speed at which SARS-CoV-2 spread around the globe took many countries and their health systems by surprise. The coronavirus pandemic presented three major difficulties, namely a surge in SARSCoV-2 infections, high mortality associated with COVID-19 disease and large patient numbers overwhelming emergency departments and intensive care units. In March 2020, the Australian government responded by establishing stand-alone fever clinics to assess patients experiencing symptoms possibly related to COVID-19. ${ }^{2}$ At a macro level, these clinics helped Australia manage many aspects of the pandemic; ${ }^{3}$ however, at a micro level, the clinics encountered various challenges. First, SARS-CoV-2 spread through the population alongside other winter-associated respiratory illnesses causing a surge in the volume of individuals experiencing COVID-19like symptoms, and thus presenting to the clinics. Second, the precautionary requirement for clinicians to don and doff personal protective equipment between patient encounters reduced patient assessment speed and efficiency. Finally, given that the symptoms of respiratory illnesses-including fever, cough, sore throat and shortness of breath ${ }^{4}$ - can be related to a number of different diseases such as COVID-19, chronic obstructive pulmonary disease (COPD), asthma, pneumonia and upper respiratory tract infections, there is diagnostic complexity in distinguishing patients with a particular respiratory disease and even more so for those with concurrent infections. ${ }^{5}$ This
Summary box

What are the new findings?

- Digital respiratory diagnostic tools developed in the pre-COVID-19 era appear to maintain diagnostic accuracy in a realworld setting, even with COVID-19 acting as a potential confounding factor.

- The implementation of digital diagnostic tools might aid overwhelmed clinics in managing patient load and in managing the exposure of clinical staff to SARS-CoV-2.

- ResAppDx is a safe, efficient and effective tool in the real-world specialist respiratory clinic environment and showed utility as an added safety-net in detecting respiratory disease in challenging clinical presentations.

How might it impact on healthcare in the future?

- Effective digital diagnostic tools can be easily adopted by overwhelmed health providers and create significant impact quickly.

- Digital tools can offer the ability for asynchronous and rapid care delivery allowing more efficient use of clinical staff, reduced need for further testing and increased patient throughput.

final challenge is exaggerated in highthroughput clinical environments, such as COVID-19 fever clinics.

A single Federal Government funded COVID-19 fever clinic in Queensland, Australia, looked to virtual health technologies as a potential way to alleviate these problems. One technology in particular-ResAppDx ('the device')—offered noticeable value to the COVID-19 fever clinic due to its ability to rapidly identify 
Table 1 ResAppDx select clinical diagnosis list and clinical efficacy data

\begin{tabular}{lll}
\hline & Positive percent agreement/sensitivity & Negative Percent Agreement/specificity \\
\hline Lower respiratory tract disease (>12 years old) & $88 \%(95 \% \mathrm{Cl}, 84 \% \text { to } 91 \%)^{7}$ & $89 \%(95 \% \mathrm{Cl}, 83 \% \text { to } 93 \%)^{7}$ \\
\hline Pneumonia (>12 years old) & $86 \%(95 \% \mathrm{Cl}, 80 \% \text { to } 91 \%)^{8}$ & $87 \%(95 \% \mathrm{Cl}, 80 \% \text { to } 91 \%)^{8}$ \\
Asthma exacerbation (>12 years old) & $89 \%(95 \% \mathrm{Cl}, 76 \% \text { to } 96 \%)^{9}$ & $84 \%(95 \% \mathrm{Cl}, 73 \% \text { to } 91 \%)^{9}$ \\
$\begin{array}{l}\text { Chronic obstructive pulmonary disease } \\
\text { exacerbation (>12 years old) }\end{array}$ & $83 \%(95 \% \mathrm{Cl}, 73 \% \text { to } 90 \%)^{10}$ & $91 \%(95 \% \mathrm{Cl}, 82 \% \text { to } 96 \%)^{10}$ \\
Croup & $88 \%(95 \% \mathrm{Cl}, 78 \% \text { to } 95 \%)^{11}$ & $86 \%(95 \% \mathrm{Cl}, 82 \% \text { to } 89 \%)^{12}$ \\
Bronchiolitis (children<2 years old) & $76 \%(95 \% \mathrm{Cl}, 60 \% \text { to } 88 \%)^{11}$ & $60 \%(95 \% \mathrm{Cl}, 59 \% \text { to } 70 \%)^{11}$ \\
\hline
\end{tabular}

common respiratory diseases using fixed machine learning (ML) algorithms that analyse cough sounds. This technology-driven approach might therefore allow for asynchronous patient assessments. The device has achieved regulatory approval in Europe and Australia ${ }^{6}$ and can rapidly indicate the presence of several respiratory diseases including: lower respiratory tract disease (LRTD), pneumonia, asthma exacerbation, COPD exacerbation, croup and bronchiolitis (table 1). ${ }^{7-11}$

The device itself is a point-of-care, handheld smartphone software application that analyses acoustic patterns in a patient's cough along with simple patientreported symptoms to assist with differentiating respiratory tract illness. ${ }^{1112}$ The software application can be downloaded and installed on a range of smartphones, and the ML algorithms contained within the application have been clinically assessed in two blind prospective trials. ${ }^{13-15}$ The device used in this assessment has received CE marking and Australian Register of Therapeutic Goods listing as a Class IIa medical device. ${ }^{6}$ The device is required to be held $\sim 50 \mathrm{~cm}$ away from the patient with the microphone directed toward the patient's mouth at an approximately $45^{\circ}$ angle, laterally from the patient's mouth. This allows the device operator to keep at a distance to the patient.

The respiratory clinic sought to achieve three primary objectives through using the device: (1) aid in the detection of pneumonia-the most common complication of COVID-19 16 -as early antibiotic treatment can improve clinical outcomes ${ }^{17}$; (2) effectively evaluate patients for respiratory diseases without increasing the risk of SARS-CoV-2 transmission to clinical staff and (3) improve patient throughput by increasing the speed of patient assessment.

\section{METHODS}

This assessment was conducted at a single Federal Government funded COVID-19 fever clinic. The device was operated by a trained nurse, with the results used by two clinic physicians ('using physicians') as part of their treatment workflow. Patients were verbally consented prior to the use of the device, as it was used in its intended manner as a regulated medical device.

Data were collected between May 2020 and August 2020 ('assessment period') during clinics where one or more using physician and the trained nurse was present at the clinic. The trained nurse operated the device on a hand-held smartphone prior to physician encounters to allow for asynchronous assessment and shortened individual patient encounters. The results provided by the device were available to using physicians at the time of clinical assessment in the form of an analysis report (figure 1). For a small subcohort of patients, the stopwatch function on a second hand-held smartphone was used to record the time to diagnosis when using the device. The stopwatch was started as the

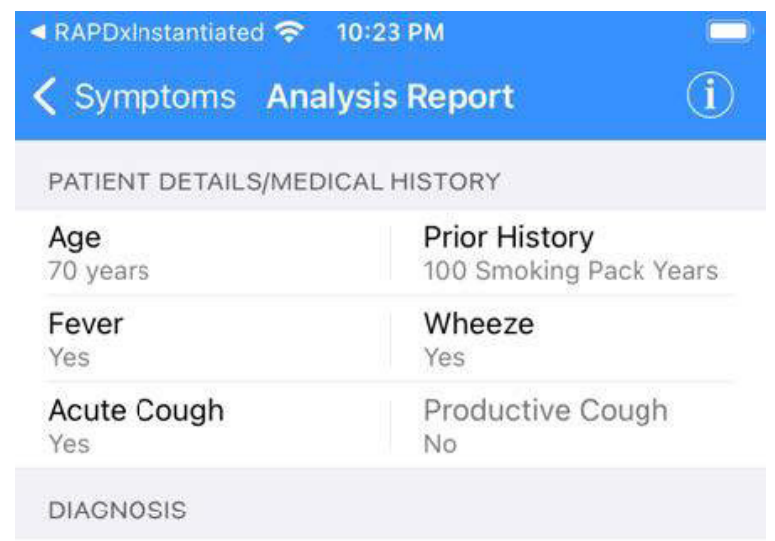

\section{Lower Respiratory Tract Disease} INDICATED

\section{Pneumonia}

INDICATED

Note: Chronic Obstructive Pulmonary Disease
(COPD) may be present.

Patient not tested for the following due to medical history: asthma exacerbation, COPD exacerbation.

CAUTION: Not for use as the basis for treatment without independent medical confirmation.

\section{Clean device before reuse}

Learn how >
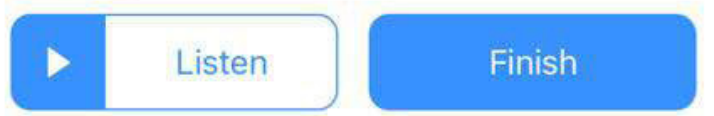

Figure 1 ResAppDx Analysis Report screen example. 
nurse began their assessment-which included patient identification and confirmation, device set-up, provision of five coughs by the patient and cough sound analysis-and was stopped at the close of the clinical assessment and provision of an analysis report.

The device can output up to six possible indications: LRTD, pneumonia, asthma exacerbation, COPD exacerbation, croup and bronchiolitis. These indications are not mutually exclusive and as such the indication for LRTD in particular-which is an umbrella indication for unspecified lower respiratory tract disease-is often identified alongside other respiratory disease indications. Using physicians used the results of the device as part of their overall clinical assessment to reach a final diagnosis, decide on the need for radiological confirmation, and commence appropriate treatment. In clinical cases where computerised tomography ('CT') was warranted, these scans were performed. All CT scans were performed after use of the device by the treating team, and the radiologists reporting these scans were blind to the results presented by the device for each patient.

Data were collected for a total of 186 patient encounters, with patients aged between 3 and 88 years all of whom were experiencing undifferentiated respiratory symptoms ('assessment cohort'). A subgroup of 21 patients from this cohort underwent further low-dose CT of the chest ('CT cohort') based on clinical need. A second subgroup of 30 patients ('treatment cohort') was analysed looking at the indication presented by the device, the agreement against full clinical assessment, and whether treatment was commenced. The data presented for the treatment cohort have been normalised to remove codiagnoses to allow for better comparison, and it should be noted that the diagnosis groups are assessments of the same groups of patients. A final subgroup of 23 patients was used to analyse the speed at which the device presented a result to the clinical team.

\section{RESULTS}

The time to final result when using the device averaged $32 \mathrm{~s}(\mathrm{n}=23,27-39 \mathrm{~s})$.

In the assessment cohort $(\mathrm{n}=186)$, the device identified 92 patients with undifferentiated LRTD, 52 with pneumonia, three with croup, 32 with asthma exacerbation, and 23 with COPD exacerbation. Overall, 49 assessments produced no finding.

In the CT cohort $(n=21)$, the device correlated with the radiological findings in $12 / 12$ patients with undifferentiated LRTD, 8/9 patients with COPD and 1/1 patient with pneumonia. In the single non-correlated COPD patient, the device indicated the presence of possible COPD exacerbation; however, there was no prior diagnosis of COPD and no evidence of COPD was reported radiologically. Of note, the patient was found to have an approximate 25 pack year history of cigarette use. Also, of note was the correct identification of pneumonia by the device in a SARS-CoV-2 positive patient where pneumonia was subsequently reported on CT as an atypical pneumonia.

In the treatment cohort $(n=30)$, the device identified 25 patients with pneumonia of whom the using physicians reached a final diagnosis and commenced treatment for pneumonia in 17 patients. For the remaining patients in the cohort, the device and using physicians were in full concordance and identified one patient with croup, three with asthma exacerbation and one with COPD exacerbation.

\section{DISCUSSION}

Given the high degree of concordance between the indications presented by the device and both the clinical and radiological diagnoses, the results of this real-world assessment suggest the device can be safely used within a specialist respiratory assessment clinic environment and provide value to clinical practice. Clinicians found the software simple to use and integral to the clinical decision-making process, this conclusion is supported by the continued use of the device in clinical care beyond the assessment period. An important result to highlight was the very slight discordance between the indication of pneumonia by the device and an ultimate pneumonia diagnosis by the using physicians. Though the sample is too small to draw strong conclusions, it does seem that the using physicians factored the result presented by the device into their clinical decision-making; however, were not swayed by the indication and felt comfortable reaching a different conclusion using their clinical judgement.

The sentiment of the using physicians were aligned with the findings of Wipf et al who displayed limited instrumental value in performing physical examination of the chest in the detection of pneumonia-sensitivity: 47\%-69\% and specificity: $58 \%-75 \%{ }^{18}$ The using physicians therefore recognised the value in obtaining additional clinical information in reaching a correct diagnosis quickly and efficiently. For comparison, the sensitivity and specificity of the device in pneumonia is $86 \%$ and $89 \%$. The using physicians received results from the device prior to interacting with patients. They therefore used the results from the device to focus clinical decision-making, thereby saving valuable time during patient encounters. As the device was used by a trained nurse prior to patients being seen by attending physicians, the exposure risk to SARS-CoV-2 for each member of clinical staff was also reduced and distributed. The device takes on average $32 \mathrm{~s}$ to deliver a result, which allows the device operator to minimise their exposure time to patients. These alterations to the typical clinic workflow that are enabled by the use of the device are important considerations not just in the context of the COVID-19 pandemic but also periods of increased pressure on health systems such as during the winter months. The authors have come to trust the device, and rely on it to maintain the efficiency of 
clinical operations and help minimise the transmission risk of SARS-CoV-2.

In detecting a COPD exacerbation in a potentially undiagnosed COPD patient and indicating the presence of pneumonia in a COVID-19-positive individual with atypical pneumonia, the device showed utility in detecting a range of clinical diagnoses that were difficult to ascertain from the initial clinical picture available to the using physicians. The use of the device thus allowed for more precise clinical care to be delivered, earlier treatment to be commenced, and worked to increase patient throughput. The device was found to have utility in detecting respiratory infections (pneumonia was detected in 25 cases in the treatment cohort which in 17 patients was confirmed with full clinical assessment) which for a number of patients allowed for early antibiotic administration by the using physicians. The device sensitivity was also assessed through correlates with low-dose CT scan results where the device correctly identified concurrent lung disease such as COPD exacerbation. Further, in the one case of discordant diagnosis between the device and CT scan, use of the device prompted further investigation and follow-up of a patient with an extensive smoking history who did not yet have CT changes associated with COPD. This finding is of particular note as the early introduction of-predominantly nonpharmacological-treatments for COPD are vital in preventing the progression of the disease. ${ }^{19}$ Anecdotally, the using physicians found that through continued use of the device and its growing concordance with CT scan in particular they grew less weary of its results and more naturally factored it into their clinical decisionmaking. The authors feel this point should be noted as an initial period of hesitance by clinicians might be expected when implementing digital tools for the first time. Lastly, as the device is operated at a distance to the patient, the use of the device allowed clinical staff to maintain a safe distance from patients during clinical encounters.

There are a number of limitations to address in how the device was used in this study and what data were gathered. First, due to regulatory requirements, there is the necessity for a clinic staff member to operate the device. The authors believe that a valuable avenue to explore is the ability to allow patients to operate the device while isolated in a clinic room prior to clinical assessment. This would further reduce the risk of viral transmission to clinical staff. Second, there are limitations inherent in the fact that this observational assessment utilises a small sample size and has been performed at a single centre. The conclusions that the use of the device improves clinical experience and displays concordance with clinical and radiological findings will need to be validated in a larger, multisite assessment. Finally, as this was a small assessment that explored the implementation and utility of a device, a comparison against a control patient population was not performed. This along with an exploration of the anecdotal findings noted by the authors such as reduced need for confirmatory radiology, and decreased movement of patients to other areas of the clinic is something that should be considered when undertaking a larger, multisite assessment.

\section{CONCLUSION}

Patients with respiratory symptoms have always presented challenges to health providers. These challenges have increased with the addition of COVID-19 and the risk of concurrent respiratory illness. The device implemented and presented here proved to be a safe, simple and effective tool in the specialist respiratory clinic environment and successfully detected a range of respiratory diseases, sped up clinical care delivery and helped lower potential SARS-CoV-2 transmission risks. We hope these findings encourage others to pilot novel digital health tools to ease longstanding clinical pressures as well as those brought on by the COVID-19 pandemic.

\section{Twitter Shrawan Patel @DrShrawanPatel}

Acknowledgements We would like to thank the team at ResApp Health Limited for their help in enabling a smooth implementation of the device into the clinical workflows at the clinic. We would also like to thank the clinical team at Health Hub Doctors Morayfield for their tireless service throughout the coronavirus pandemic, for embracing use of the device so readily and for providing invaluable feedback.

Contributors The concept and execution of this study was conceived by AL. Execution of many elements of this study were also performed by MÇ. Interpretation of the data was performed by AL and SP, and drafting of the manuscript was completed by SP with input from $\mathrm{AL}$ and $\mathrm{MÇ}$.

Funding The authors have not declared a specific grant for this research from any funding agency in the public, commercial or not-for-profit sectors.

Competing interests $\mathrm{AL}$ and MÇ have nothing to disclose. SP reports personal fees from ResApp Health, during the conduct of the study; personal fees from ResApp Health, outside the submitted work.

Patient consent for publication Not applicable.

Ethics approval This study involves human participants but was not approved by this assessment. This study was designed as an observational quality improvement project to understand and assess the use of a regulatory-approved medical device in novel clinical setting as judged by established/accepted standards. The assessment directly benefits the clinic and it does not increase risk to patients. Patients underwent assessment by the medical device as a component of care with the aim of comparing the altered workflow to established standards. Participants gave informed consent to participate in the study before taking part.

Provenance and peer review Not commissioned; externally peer reviewed.

Open access This is an open access article distributed in accordance with the Creative Commons Attribution Non Commercial (CC BYNC 4.0) license, which permits others to distribute, remix, adapt, build upon this work non-commercially, and license their derivative works on different terms, provided the original work is properly cited, appropriate credit is given, any changes made indicated, and the use is non-commercial. See: http://creativecommons.org/ licenses/by-nc/4.0/.

ORCID iDs

Andrew Ladhams http://orcid.org/0000-0001-9368-7427 
Shrawan Patel http://orcid.org/0000-0001-8181-277X

Mathew Çetin http://orcid.org/0000-0003-0976-9185

\section{REFERENCES}

1 Guan W-J, Ni Z-Y, Hu Y, et al. Clinical characteristics of coronavirus disease 2019 in China. N Engl J Med 2020;382:1708-20.

2 Australia to unveil $\$ 2.4$ bn coronavirus health package including pop-up fever clinics, 2021. Available: https://www. theguardian.com/world/2020/mar/11/australia-to-unveil-24bncoronavirus-health-package-including-pop-up-fever-clinics [Accessed 03 Jan 2021].

3 Australian Government Department of Health. Australian health sector emergency response plan for novel coronavirus (COVID-19), 2020. Available: https://www.health.gov.au/ resources/publications/australian-health-sector-emergencyresponse-plan-for-novel-coronavirus-covid-19

4 Matteelli A, Saleri N. Respiratory diseases. Travel Medicine 2008:561-72.

5 Ginsburg AS, Klugman KP. COVID-19 pneumonia and the appropriate use of antibiotics. Lancet Glob Health 2020;8:e1453-4.

6 Australian government department of health. Australian government department of health therapeutic goods administration, 2021. Available: https://www.ebs.tga.gov. au/servlet/xmlmillr6?dbid=ebs/PublicHTML/pdfStore.nsf \& docid $=$ 9D7387C5E530A256CA25848600421CB3\&agid $=($ PrintDetailsPublic)\&actionid=1 [Accessed 20 Nov 2021].

7 Porter P, Claxton S, Brisbane J. Diagnosis of lower respiratory tract disease (lrtd) and pneumonia using a smartphone-based cough-centred algorithm in an adolescent and adult acute-care cohort. Respirology 2019;24:23 https://espace.library.uq.edu. au/view/UQ:e1c1e19

8 Porter P, Brisbane J, Abeyratne U, et al. Diagnosing community-acquired pneumonia via a smartphone-based algorithm: a prospective cohort study in primary and acutecare consultations. Br J Gen Pract 2021;71:e258-65.

9 Claxton S, Porter P, Brisbane J. Detection of asthma exacerbation in adolescent and adult subjects with chronic asthma using a cough-centred, smartphone-based algorithm. Respirology 2020;25:111-230.

10 Porter P, Claxton S, Wood J. Late Breaking Abstract Diagnosis of chronic obstructive pulmonary disease (COPD) exacerbations using a smartphone-based, cough-centred algorithm. Eur Respir J 2019;54:PA4278 https://www.erseducation.org/lr/show-details/?idP=238062
11 Porter P, Abeyratne U, Swarnkar V, et al. A prospective multicentre study testing the diagnostic accuracy of an automated cough sound centred analytic system for the identification of common respiratory disorders in children. Respir Res 2019;20:81-6.

12 Porter P, Claxton S, Brisbane J, et al. Diagnosing chronic obstructive airway disease on a smartphone using patientreported symptoms and cough analysis: diagnostic accuracy study. JMIR Form Res 2020;4:e24587.

13 NHMRC Clinical Trials Centre, University of Sydney (Australia). Australian New Zealand Clinical Trials Registry [Internet]: Sydney (NSW); 2005 -. Identifier ACTRN12620000144910. A Pilot Study using Non-Contact Smartphone Cough Sound Recordings to Screen for Chronic Obstructive Pulmonary Disease in an Indigenous Australian Adult Population, 2020. Available: http://www.anzctr.org.au/ ACTRN12620000144910.aspa [Accessed 20 Dec 2020].

14 NHMRC Clinical Trials Centre, University of Sydney (Australia). Australian New Zealand Clinical Trials Registry [Internet]: Sydney (NSW); 2005 -. Identifier ACTRN12618001521213. The Breathe Easy Study: Developing digital diagnostic tests for respiratory diseases using non-contact sound recordings in children and adults. Initial technical training and development phase and secondary blinded prospective diagnostic accuracy studies, 2018. Available: http://www.anzctr.org.au/ACTRN12618001521213. aspa [Accessed 20 Dec 2020].

15 ClinicalTrials.gov. Bethesda (MD): National library of medicine (US). 2000 Feb 29 -. identifier NCT03392363, diagnosing respiratory disease in children using smartphone recordings of cough sounds 2; 2017 Dec 29, 2017. Available: https://www. clinicaltrials.gov/ct2/show/NCT03392363 [Accessed 20 Dec 2020].

16 Grippo F, Navarra S, Orsi C, et al. The role of COVID-19 in the death of SARS-CoV-2-Positive patients: a study based on death certificates. J Clin Med 2020;9:3459.

17 Houck PM, Bratzler DW, Nsa W, et al. Timing of antibiotic administration and outcomes for Medicare patients hospitalized with community-acquired pneumonia. Arch Intern Med 2004;164:637-44.

18 Wipf JE, Lipsky BA, Hirschmann JV, et al. Diagnosing pneumonia by physical examination: relevant or relic? Arch Intern Med 1999;159:1082-7.

19 Global Initiative for Chronic Obstructive Lung Disease (GOLD). Global strategy for the diagnosis, management, and prevention of chronic obstructive pulmonary disease, 2020. Available: www.goldcopd.org2020 